\title{
Correction to: Combined resistance and aerobic exercise training reduces insulin resistance and central adiposity in adolescent girls who are obese: randomized clinical trial
}

\author{
Leena P. Bharath ${ }^{1,2}$. William W. Choi ${ }^{1}$. Jae-min Cho ${ }^{4}$. Alexus A. Skobodzinski ${ }^{1}$ Alexei Wong ${ }^{5} \cdot$ Ty E. Sweeney $^{1}$. \\ Song-Young Park ${ }^{2,3}$
}

Published online: 5 January 2021

(c) Springer-Verlag GmbH Germany, part of Springer Nature 2020

\section{Correction to: \\ European Journal of Applied Physiology \\ (2018) 118:1653-1660 \\ https://doi.org/10.1007/s00421-018-3898-8}

The incorrect IRB number under "Participants" section was accidently reported. The text was incorrect and should have no IRB\#, since this study was performed in 2009. South Korea's IRB was initiated in 2013, therefore, an approved IRB was not required to carry out these research procedures. However, data collection and all the procedures were appropriately performed in accordance with the Declaration of Helsinki, and informed consent from each participant was still received despite no IRB approval requirements for study initiation. Therefore, there were no ethical violations or issues for the study. We apologize for this error.

Publisher's Note Springer Nature remains neutral with regard to jurisdictional claims in published maps and institutional affiliations.
The original article can be found online at https://doi.org/10.1007/ s00421-018-3898-8.

Song-Young Park

Song-youngpark@unomaha.edu

1 Department of Microbiology, Boston University School of Medicine, Boston, MA, USA

2 Department of Physical Education, Pusan National University, Busan, South Korea

3 School of Health and Kinesiology, University of Nebraska, Omaha, 6001 Dodge Street, Omaha, NE 68182, USA

4 Department of Nutrition and Integrative Physiology, University of Utah, Salt Lake City, UT, USA

5 Department of Health and Human Performance, Marymount University, Arlington, VA, USA 